九州大学学術情報リポジトリ

Kyushu University Institutional Repository

Further Studies on Histochemical and Ultrastructural Properties of the Pectoral Muscles of Bats

Ohtsu, Ryuichi

Zoological Laboratory, Faculty of Agriculture, Kyushu University

Uchida, Teruaki

Zoological Laboratory, Faculty of Agriculture, Kyushu University

https://doi.org/10.5109/23704

出版情報：九州大学大学院農学研究院紀要. 24 (2/3)，pp. 145-155，1979-11. Kyushu University バージョン：

権利関係 : 


\title{
Further Studies on Histochemical and Ultrastructural Properties of the Pectoral Muscles of Bats
}

\author{
Ryuichi Ohtsu and Teru Aki Uchida \\ Zoological Laboratory, Faculty of Agriculture, \\ Kyushu University 46-06, Fukuoka 812 \\ (Received August 6, 1979)
}

\begin{abstract}
The pectoral muscles (anterior division) of two bat species with different modes of flight were investigated histochemically and ultrastructurally. According to the histochemical study, the pectoral muscles of Miniopterus schreibersi fuliginosus which can fly a long distance with speedy and enduring flights were constituted with two muscle fiber types (mitochondria-more rich and mitochondria-moderate fibers), while those of Rhinolophus ferrumequinumnippon which can fly slowly and delicately were composed of three muscle fiber types (mitochondria-more rich, mitochondria-less rich and mitochondria-moderate fibers). As to the distinctive ultrastructural features of the three muscle fiber types, sarcoplasmic reticulum and the folds of neuromuscular junction were more developed in the mitochondria-more rich and mitochondria-moderate fibers of both species than in the mitochondrialess rich fiber. It is concluded that there is a correlation among fiber composition and ultrastructural features, and physiological properties of the pectoral muscles needed for different types of flight.
\end{abstract}

\section{INTRODUCTION}

The results obtained from histochemical, physiological and biochemical (Barnard et al., 1971; Peter et al., 1972) and electron microscopical studies (Gauthier, 1969) suggest the existance of three fiber types in mammalian skeletal muscles. Further, a number of experiments revealed a close relationship between muscle function and fiber type composition (reviewed by Close, 1972; Burke and Edgerton, 1975). In a previous paper (Ohtsu et al., 1978), we reported an apparent metabolic adaptation of bats' pectoral muscles.

The purpose of the present investigation is to determine fiber composition, and to describe the structure of the sarcotubular system and neuromuscular junction in the pectoral muscles of both M. S. fuliginosus, which is an enduring flier (a narrow and long wing-type) and R.f. nippon, which takes only short flights (a wide and short wing-type), with the aim of correlating structure with function from the standpoint of adaptation for flight.

\section{MATERIALS AND METHODS}

The bats used in this study were adult males of two species, Miniopterus schreibersi fuliginosus (body weight ca. 13g) and Rhinolophus ferrumequinum nippon 
(ca. $23 \mathrm{~g}$ ), collected at Ohse-db Cave, Kumamoto Prefecture, Japan in July. The muscles used here were of the superficial middle portion of the pectoral muscles (anterior division), since they are the most massive and important muscles controlling the downstroke of wings in bats.

For the histochemical examination, the muscles were immediately frozen in liquid nitrogen, then transferred into a cryostat and cut transversely into $10 \mu \mathrm{m}$ sections. Sections were stained for succinic dehydrogenase (SDH) activity by the method of Nachlas et al. (1957).

For the electron microscopical examination, the muscles were fixed in $3 \%$ glutaraldehyde buffered with $0.12 \mathrm{M}$ sodium cacodylate at $\mathrm{pH} 7.2$ for $2 \mathrm{hr}$, then washed briefly in the same buffer and further fixed for $2 \mathrm{hr}$ in $1 \%$ osmium tetroxide buffered with sodium cacodylate at $\mathrm{pH} 7.2$ with ruthenium red (Luft, 1970) or without ruthenium red. Most samples were stained en bloc with $1 \%$ uranyl acetate in aqueous solutions before dehydration. The tissues were dehydrated in alcohol series and embedded in Epon 812. Thin sections $(600 \mathrm{~nm})$ for electron microscopy were cut with glass knives on a Porter-Blum MT-1 microtome, and stained with lead acetate or doubly stained with lead and uranyl acetate. The sections were examined with a Hitachi HS-9 electron microscope.

\section{RESULTS}

\section{Histochemical observations}

In the sections incubated for succinic dehydrogenase, two types of muscle fiber, i.e., a strongly positive type with small diameter (mitochondria-more rich fiber: high oxidative fiber) and a weakly positive type with large diameter (mitochondria-moderate fiber: low oxidative fiber), are found in $M$. s. fuligixosus (Fig. 1A). On the other hand, three types of muscle fiber, i.e., a

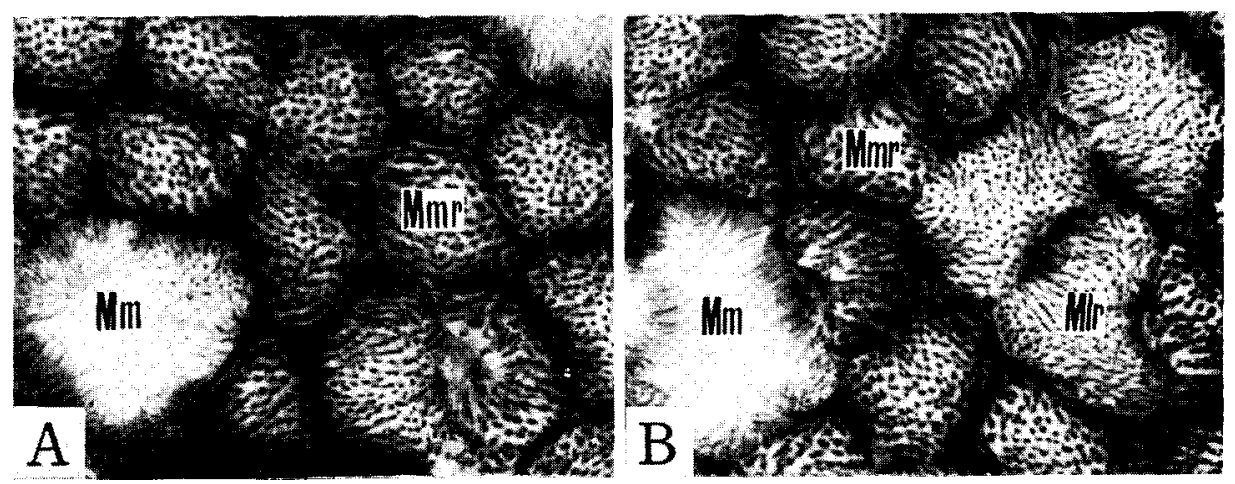

Fig. 1. Cryostat sections of the pectoral muscles of bats stained for S D H (A, B). Two types of muscle fiber in M.s.fuliginosus (A); three types of muscle fiber in R. f.nippon (B). Mlr, mitochondria-less rich fiber; $\mathrm{Mm}$, mitochondria-moderate fiber; Mmr, mitochondria-more rich fiber. x 700 . 
strongly positive type with small diameter (mitochondria-more rich fiber), a positive type with intermediate diameter (mitochondria-less rich fiber : moderate oxidative fiber) and a weakly positive type with large diameter (mitochondria-moderate fiber), are recognized in $R$. $f$. nippon (Fig. 1B).

The distribution of the different fibers is not uniform throughout the pectoral muscles of these bats. That is, the mitochondria-more rich fibers occur in greater concentration in the deep area of the pectoral muscles, while the mitochondria-less rich and mitochondria-moderate fibers are predominant in the superficial part.

\section{Electron microscopic observations}

Sarcotubular system

From the result of exposure to ruthenium red (Fig. 2), it is known that a transverse tubule (T-tubule) communicates with the extracellular space in the pectoral muscles of these bats.

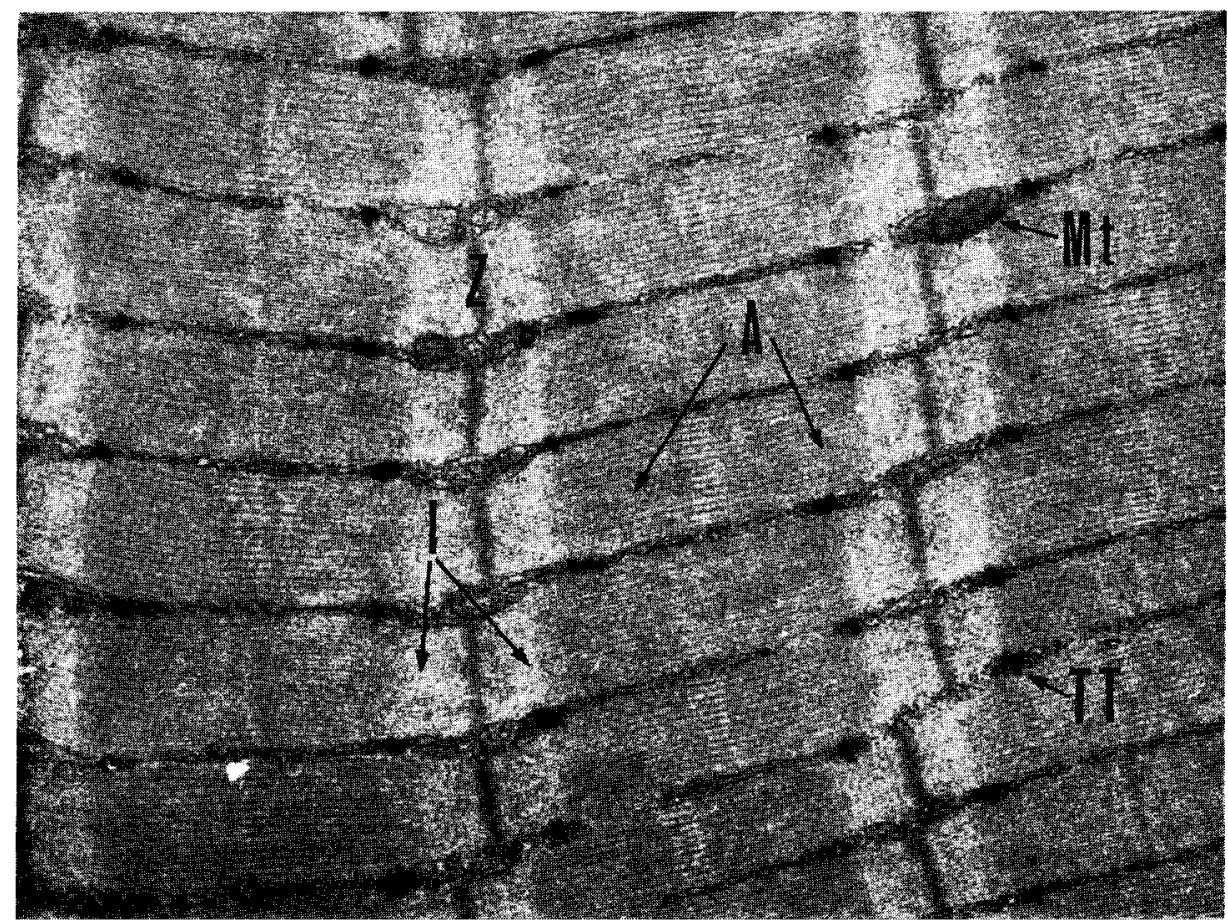

Fig. 2. Electron micrograph of the mitochondria-moderate fiber of the pectoral muscles in M. s. fuliginosus. An accumulation of ruthenium red is recognizable in the transverse tubule (TT). A, A-band; I, I-band; Mt, mitochondria; $Z$, Z-line. $\times 25,000$.

The distribution of sarcoplasmic membrane system in the different muscle fiber types appears to resemble each other; that is, as shown in Fig. 3, triads occur at the $\mathrm{A}-\mathrm{I}$ junctions, and longitudinal tubules extend from terminal cis- 
ternae toward the center of the A-band, forming a more or less transverse network and sometimes a fenestrated collar. The T-tubule is closely associated with lateral sacs of the sarcoplasmic reticulum. The junction between T-tubule and terminal cisternae is complex and forms the so-called "SR feet". Longitudinal prolongations of the junctional cisternae are frequently filled with characteristic granular materials. The sarcoplasmic reticulum between the triads in the region of the $Z$-line consists generally of an interwining meshwork of tubules which sometimes contains fenestrations similar to the fenestrated collar in the A-band. The triads are closely aligned to the mitochondria that encircle myofibrils at the I-bands (Fig. 3 inset).

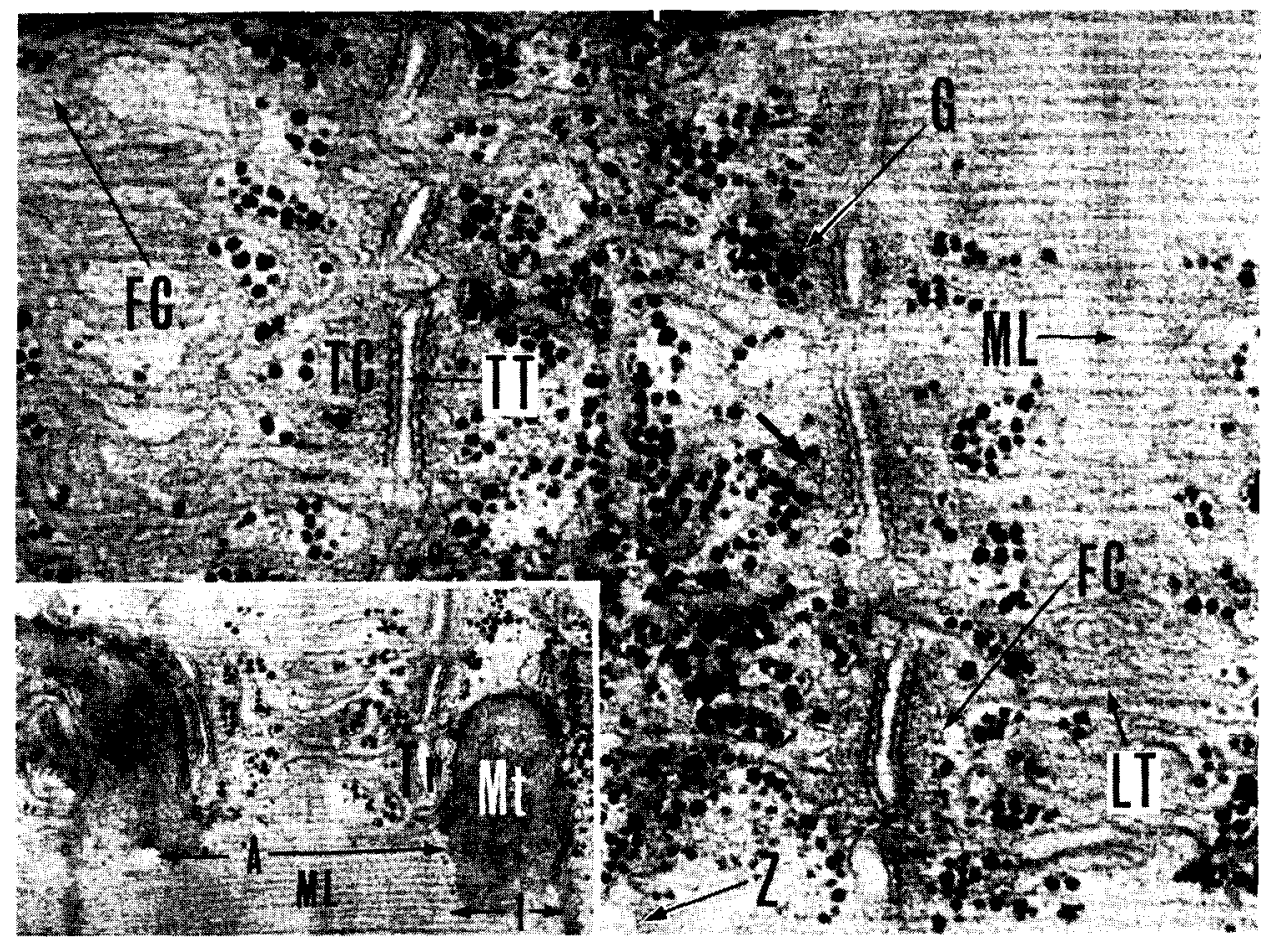

Fig. 3. Longitudinal section illustrating organization of sarcotubular system of the mitochondria-moderate fiber of the pectoral muscles in M.s. fuliginosus. The longitudinal tubules (LT) are merged into the fenestrated collar (FC) not only at the mid-sarcomere level, but also near the junctional cisternae. Z-line reticulum has fenestrations (arrow). A, Aband; G, glycogen granule; I, I-band; ML, M-line; Mt. mitochondria; TC, terminal cisternae; Tr, triad; TT, transverse tubule; Z, Z-line. $\mathrm{x} 50,000$. Inset $\times 25,000$

Differences, however, in the degree of development of sarcoplasmic reticulum exist among the fiber types, that is, the mitochondria-more rich (Figs. $4 A$ and $5 A$ ) and mitochondria-moderate (Figs. $4 B$ and $5 C$ ) fibers of both species have a well developed sarcoplasmic reticulum, while the mitochondria- 

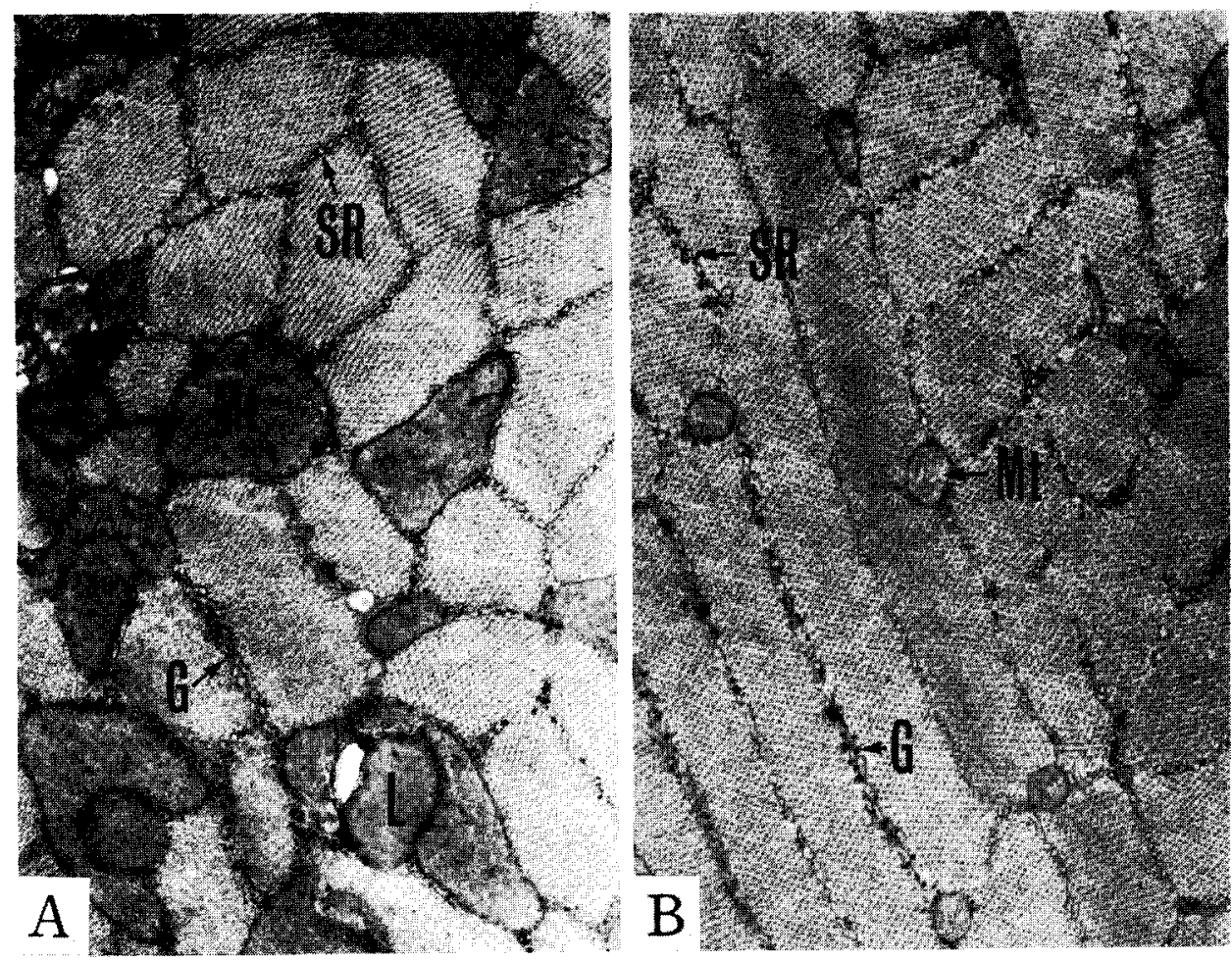

Fig. 4. Transverse sections of the two fiber types of the pectoral muscles in M.s. fuliginosus. In the mitochondria-more rich (A) and mitochondria-moderate (B) fibers, a single or double layers of sarcoplasmic reticulum (SR) flanked by glycogen granules (G) encircle regularly the myofibrils. L, lipid droplet; Mt, mitochondria. 220,000 .

less rich fiber (Fig. 5B) of $R$. f . nippon have a less developed sarcoplasmic reticulum.

\section{Neuromuscular junction}

In all the fiber types of the pectoral muscles, branches of the motor nerve fibers terminate in an ultrastructural arrangement which is, in general, typical of neuromuscular junction as elsewhere. In the terminal axoplasm, moderate numbers of vesicles and mitochondria occur. Sarcoplasmic mitochondria are numerous in the junctional area, nuclei being aggregated in the sarcoplasm of the neuromuscular junction. However, junctional folds of the mitochondria-more rich fiber (Fig. 6A) are less closely spaced than are those of the mitochondria-moderate fiber (Fig. 6C), yet even longer than are those of the mitochondria-less rich fiber (Fig. 6B) ; in the mitochondria-less rich fiber junctional folds are relative shallow and irregular; in the mitochondriamoderate fiber regular junctional folds are longest and most numerous.

\section{Width of the Z-line}

Z-lines are widest in the mitochondria-more rich fiber (Fig. 7A), interme- 

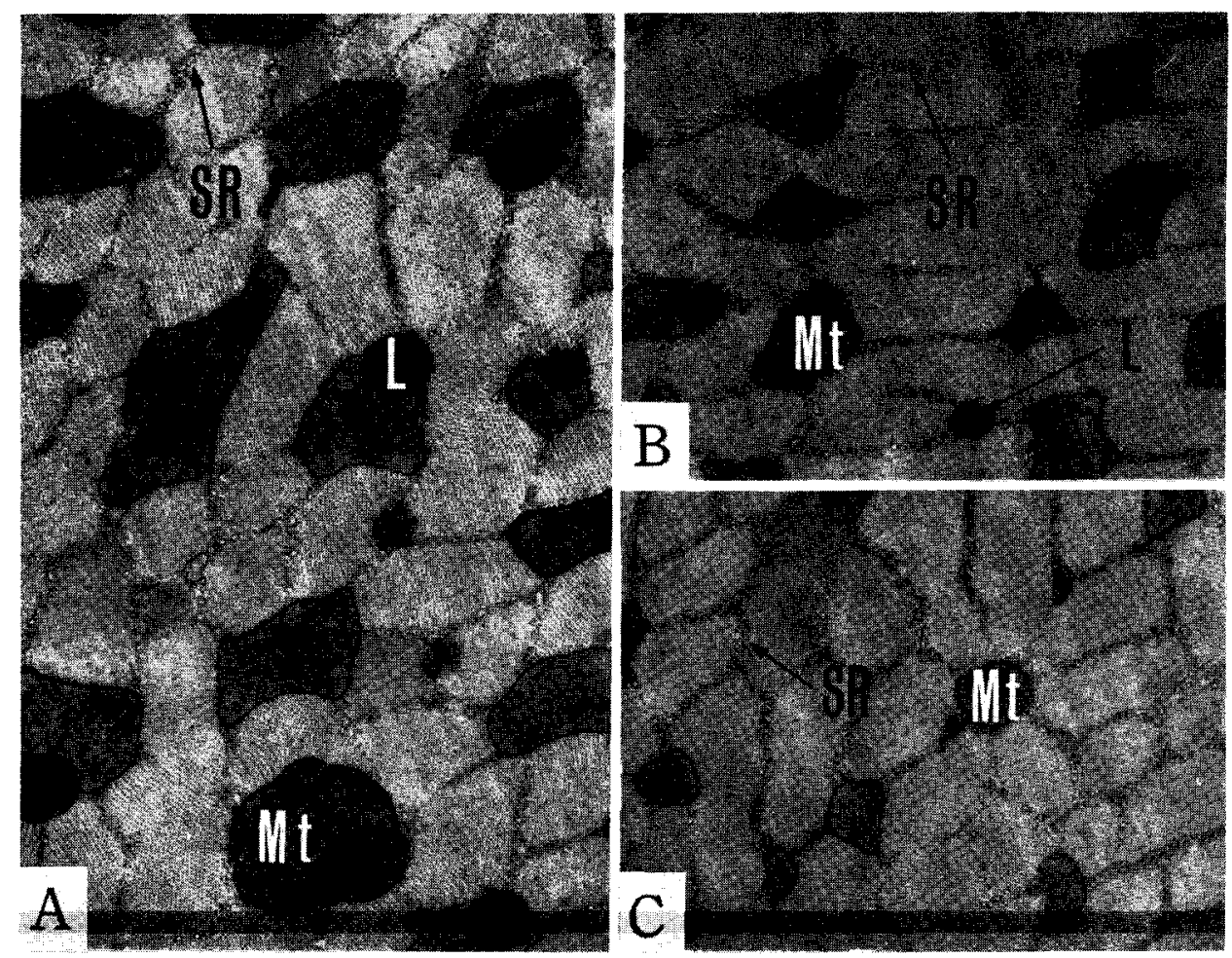

Fig. 5. Transverse sections of the three fiber types of the pectoral muscles in $R$. $f$. nippon. The mitochondria-more rich (A), mitochondrialess rich (B) and mitochondria-moderate (C) fibers are represented. L, lipid droplet; Mt, mitochondria ; SR, sarcoplasmic reticulum. x 12,500.

diate in the mitochondria-less rich fiber (Fig. 7B), and narrowest in the mitochondria-moderate fiber (Fig. 7C).

\section{DISCUSSION}

Structure of the pectoral muscles

According to SDH staining pattern, the pectoral muscles of these bats contained fibers with histochemically different staining intensities as well as both acromiotrapezius and acromiodeltoideus muscles of bat (Armstrong et al., 1977).

The assumption that both mitochondria-more rich and mitochondria-moderate fibers in the pectoral muscles of these bats are fast-twitch fibers, also, is consistent with the comparably rich development of the sarcoplasmic reticulum in these muscle fibers. On the other hand, in the mitochondria-less rich fiber the development of the sarcoplasmic reticulum was less prominent. The varied degrees of development of the sarcoplasmic reticulum appears thus to be the only ultrastructurally demonstrable feature which bears a re- 

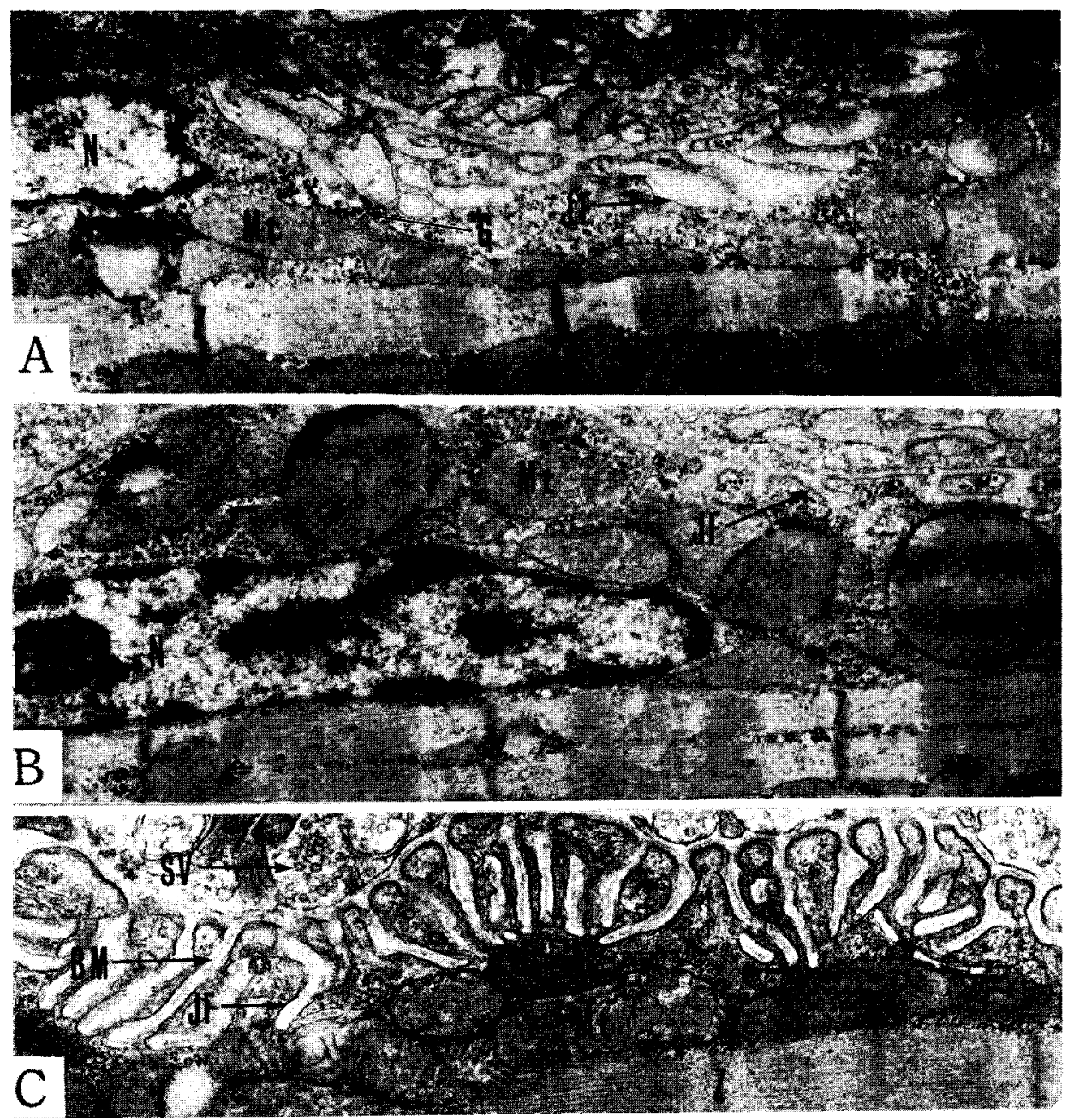

Fig. 6. Neuromuscular junction from the three fiber types of the pectoral muscles of bats. The mitochondria-more rich (A) fiber of M.s. fuliginosus, and the mitochondria-less $\operatorname{rich}(\mathrm{B})$ and mitochondria-moderate (C) fibers of $R$. f.nippon are shown. BM, basement membrane; G, glycogen granule; JF, junctional fold; L, lipid droplet; Mt, mitochondria; $\mathrm{N}$, nucleus; SV, synaptic vesicle ; $Z$, Z-line. $\times 15,000$.

lationship to the speed of contraction.

The terminal cisternal portion of the reticulum has been implicated in the release of calcium at the time of depolarization. The interconnected fenestrated tubules, however, have not been known to have a specific function. One might suspect that they function in the uptake of calcium during relaxation. The presence of fenestrations indicates that this structure acts on the rapid release or uptake of some materials. Frank (1964) suggested that extra- 


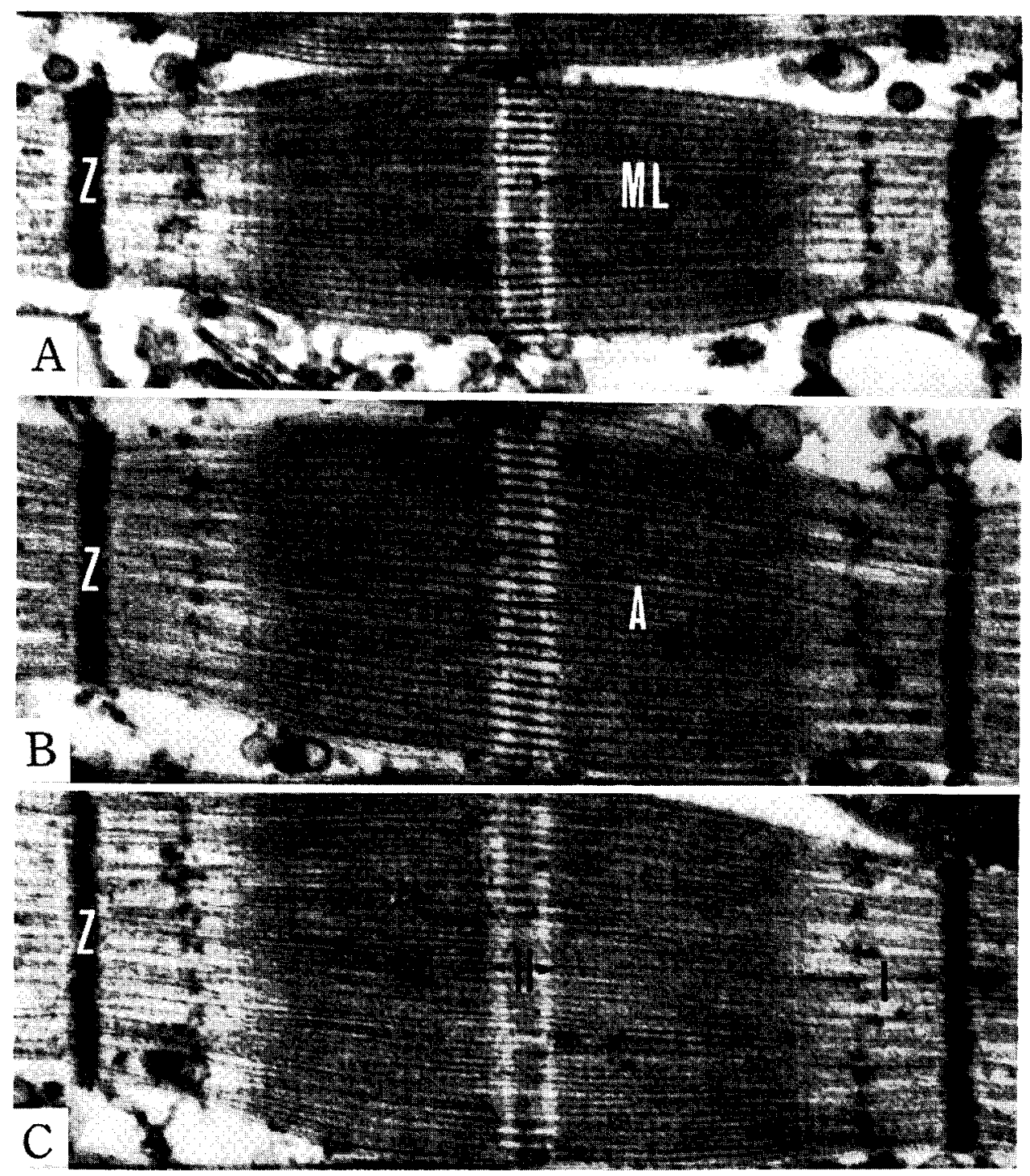

Fig. 7. Comparison of the width of the Z-line among the three fiber types of the pectoral muscles of bats. The mitochondria-more rich (A) fiber of M.s.fuliginosus, and the mitochondria-less rich (B) and mitochondria-moderate (C) fibers of R. f. nippon are indicated. A, A-band; H, H-zone ; I, I-band ; ML, M-line ; Z, Z-line. 50,000.

cellular calcium was required for muscle contraction, and there were some evidences for mitochondria being accumulation sites for calcium in muscle (summarized by Weber, 1966). Apparent connections between the sarcotubular system and mitochondria were observed by Hikida (1972). If this is true, 
the extracellular calcium may be taken into the muscle through the T-tubule and shuttled into the mitochondrion. This calcium would then be released during depolarization, or would provide the reticulum with more calcium if required. This discussion relating mitochondria and calcium brings forward the premise that the mitochondria and part of the sarcotubular system are in contact or actually connected. The contact of mitochondria with the sarcotu. bular system was recognized in the pectoral muscles of these bats, which might indicate that mitochondria act as accumulation sites for calcium.

Chinoy and George (1965), who studied the cholinesterase activity at the end plates in the pectoral muscles of frog, reptiles, wild rat and several birds, found that all end plates in twitch fibers were of the "en plaque" (focally innervated) type. In the light of these observations they proposed that the "en plaque" type of end plate was characteristic of the fibers of the pectoral muscles in the tetrapodan vertebrates.

In the twitch muscles of rat, three types of motor units were identified and characterized as fast, intermediate and slow in response (Close, 1967). It seems to be logical that such physiologically significant differences are reflected in structural disparities of the neuromuscular junction.

Padykula and Gauthier (1970) demonstrated that the neuromuscular junction of red, white and intermediate fibers of the rat diaphragm could be distinguished by differences in the shape and size of axonalendings and number of axoplasmic vesicles, by the distribution and spacing of the junctional folds, and by the appearance of both axoplasmic and sarcoplasmic mitochondria. According to their observations, on red fibers the axonal endings were associated with clusters of junctional folds that were relatively shallow, sparse and irregular; on white fibers the junctional folds were deeper and more numerous, and the folds occupied a more continuous and widespread area of the sarco. plasmic surface; thus at the neuromuscular junction there was a greater sarcoplasmic surface area in white than in red fibers. Judging from our observations, it is possible to conclude that the mitochondria-more rich and mitochondria-moderate fibers of these bats' pectoral muscles correspond to the fast-twitch fibers, while the mitochondria-less rich fiber is comparable to the slow-twitch fiber.

From comparison of the width of Z-lines in carefully orientated sections among the different muscle fiber types, it is revealed that the difference in width is utilized as a reliable criterion for distinguishing fiber types in the pectoral muscles of bats as well as in rat skeletal muscles (Gauthier, 1969).

Correlation among structural and biochemical properties of the pectoral muscles and ecological aspects

Barnard et al. (1971), who studied the relationships between histochemical properties, actomyosin and myosin ATPase activity, and the contractile properties of mammalian skeletal muscles, concluded that there were only two populations of fiber types, i.e., fast and slow twitch. Fast-twitch fibers might further be classified as red and white according to the mitochondrial content and other features (Gauthier, 1969). In a previous paper (Ohtsu et al., 1978), 
wc reported that the pectoral muscles of these two bats were constituted with mitochondria-rich and mitochondria-moderate fibers, and we could not observe the typical white fiber (mitochondria-poor fiber). The mitochondriamore rich fiber of both species corresponds to mitochondria-rich fiber with more rich mitochondrial content. We noticed also that mitochondria-rich fiber in $\boldsymbol{R}$. f.nippon possessed high oxidative capacities with a little variability from the mitochondria-more rich fibers; accordingly, the fiber that we regarded as the mitochondria-less rich fiber in $\boldsymbol{R}$. f.nippon is equivalent to mitochondria-rich fiber with less rich mitochondrial content.

The absence of mitochondria-poor (white) fiber in these bats, in comparison with mammalian skeletal muscles, is caused by that bats are steady-state fliers and would have relatively less need for anaerobic muscle work. By virtue of the nature of the intracellular organization adapted for aerobic metabolism which is necessary for prolonged activity, there is no doubt that the mitochondria-more rich fibers are used for sustained activity, whereas the mitochondria-moderate fibers, whose intracellular organization is adapted for anaerobic metabolism, are conductive to quick and short bursts of activity as described in rat skeletal muscles by Schiaffino et al. (1970). The mitochondrialess rich fibers, in comparison with the mitochondria-more rich fibers, may be related to the lower energy expenditure in maintaining tension with low actomyosin ATPase activity.

Bat pectoral muscles are specifically adapted for generating power during the downstroke of the wings, which is the basic movement in flight and provides most of the vertical lift. The properties of the fibers in the pectoral muscles of bats are thus well-suited to the specific demands of flight. Bats produce most of the required contractile force with one muscle during the flight. It is not surprising, therefore, that these bats' skeletal muscle fibers display the extreme degree of specialization. Comparing the pectoral muscles of $\boldsymbol{M}$. s. fuliginosus with those of $\boldsymbol{R}$. $f$. nippon, mitochondria were more developed and myoglobin concentrations were higher in the former than in the latter (Ohtsu et al., 1978). The above-mentioned differences in the properties of the fibers seem to be closely correlated to the fact that $\boldsymbol{M}$. $\boldsymbol{s}$. fuliginosus is an enduring flier, while $\boldsymbol{R}$. f. nippon is a short flier and desires a high maneuverability.

In conclusion, we suggest that the structure of bat pectoral muscles is an expression of two main functional parameters, i. e., speed of contraction and resistance to fatigue, the latter being presumably a measure of adaptation to continuous or sustained activity. Each of these factors appears to affect selectively distinct morphological characteristics of the muscle cell. The assumption that these two functional parameters in bat pectoral muscles are independently variable leads to the prediction that diverse combinations of structural features may be found in various kinds of fibers of the skeletal muscles as an adaptation for differing functional demands related to mode of flight. 


\section{ACKNOWLEDGEMENTS}

We are much indebted to the staff of Laboratory of Animal Husbandry II, Faculty of Agriculture, Kyushu University, for their facilities for histochemical examination, and to Professor E. W. Jameson, Jr. of the University of California for comments on the manuscript.

\section{REFERENCES}

Armstrong-, R. B., C. D. Ianuzzo and T. H. Kunz 1977 Histochemical and biochemical properties of flight muscle fibers in the little brown bat, Myotis lucifugus. J. Comp. Physiol., $119: 141-154$

Barnard, R. J., V. R. Edgerton, 'T.Furukawa and J. B. Peter 1971 Histochemical, biochemical and contractile properties of red, white and intermediate fibers. Amer. J.Physioz., 220: 410-414

Burke, R. E. and V. R. Edgerton 1975 Motor unit properties and selective involvement in movement. Exercise and Sport Sci. Rev., 3: 31-81

Chinoy, N. J. and J. C. George 1965 Cholinesterases in the pectoral muscle of some vertebrates. J. Physiol., Lond., 177: 346-354

Close, R. 1967 Properties of motor units in fast and slow skeletal muscles of the rat_. $J$. Physiol., Lond., 193: 45-55

Close, R. 1972 Dynamic properties of mammalian skeletal muscles. Physiol. Rev., 52: 129-197

Frank, G. B. 1364 Evidence for an essential role for calcium in excitation-contraction coupling in skeletal muscle. Proc. Roy. Soc., Lond., 160: 504-511

Gauthier, G. F. 1969 On the relationship of ultrastructural and cytochemical features to color in mammalian skeletal muscle. Z. Zellforsch., 95: 462-482

Hikida, R. S. 1972 The structure of the sarcotubular system in avian muscle. Amer. J. Anat., 134: 481-496

Luft. J. H. 1971 Ruthenium red and violet. 1. Chemistry, purification, methods of use for electron microscopy and mechanism of action. Anat. Rec., 171: 347-368

Nachlas, M. M., K. Tsnu, E. De Souza, C. Cheng and A. M. Seligman 1957 Cytochemical demonstration of succinic dehydrogenase by the use of p-nitrophenyl substituted ditetrazole. J. Histochem. Cytochem., 5: 420-436

Ohtsu, R., T.Mōri and T. A. Uchida 1978 Electron microscopical and biochemical studies of the major pectoral muscles of bats. Comp. Biochem. Physiol., 61(A) : 101-107

Padykula, H. A. and G. F. Gauthier 1970 The ultrastructure of the neuromuscular junctions of mammalian red, white and intermediate skeletal muscle fibers. J. Cell Biol., 46: $27-41$

Peter, J. B., R. J. Barnard, V. R. Edgerton, C. A. Gillespie and K. E. Stempel 1972 Metabolic profiles of three fiber types of skeletal muscle in guinea pigs and rabbits. Biochemistry, 11: 2627-2633

Schiaffino, S., V. Hanzlikova and S. Pierobon 1970 Relations between structure and function in rat skeletal muscle fibers. J. Cell Biol., 47:107-119

Weber, A. 1966 Energized calcium transport and relaxing factors. In "Current Topics in Bioenergetics," Vol. I, ed. by D. R. Samadi, Academic Press, New York and London, pp. 203-254 